\title{
Comparison of Three Different Styles of Submarine Sails on Electromagnetic Scattering under the Detection of Airborne Radar
}

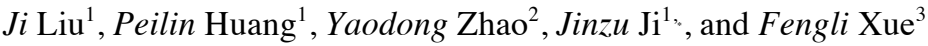 \\ ${ }^{1}$ Scholl of Aeronautic Science and Engineering, Beihang University, Beijing, 100191, China \\ ${ }^{2}$ Science and Technology on Electronic Information Control Laboratory, Chengdu, 610036, China \\ ${ }^{3}$ Key Lab for Information Science of Electromagnetic Waves (MoE), Fudan University, Shanghai \\ 200433, China
}

\begin{abstract}
This paper studies radar cross section of submarine sails on the water. Under the detection of the enemy's airborne radar, considering the motion of the submarine, the sail model of radar pitch angle incidence range analysis is established. By using CATIA software, the 3D models of AKULA sail, SUBOFF sail and VICTOR sail are built. On the basis of the physical optics method and the equivalent currents method, the scattering characteristics of sails RCS(radar cross section) are simulated under X radar band. Through the microwave anechoic chamber test, this paper verifies the accuracy of the combination of the physical optics method and the equivalent electromagnetic flow method. The influence of the distance from the airborne radar to the sail on the pitch angle of the electromagnetic wave is discussed, with the elevation angle of the radar antenna varies. Then, we illustrate the characteristics of circumferential direction RCS of the sail under different pitch angles. Finally, the mean RCS of the sail at a given pitch angle is simulated. The results show that the AKULA sail is considerably superior to SUBOFF sail and VICTOR sail on stealth performance with the pitch angle less than $4^{\circ}$. But when the pitch angle exceeds $10^{\circ}$, the SUBOFF sail can be given priority.
\end{abstract}

\section{Introduction}

A good stealth performance is significant for the submarines to survive. Most of the submarines, especially conventional submarines, often need to regularly surface and ventilate. Periscope, vent valve and other devices of the submarine are often exposed in the radar wave in the depth of cruising. These devices are mostly cylindrical that make radar screen appear stable and bright display. It can be seen that radar is still one of the important means of detecting submarines, which need to be considered its radar stealth performance.

The sail is mainly used for enclosing pressure command, lock chamber and various lifting devices, which is an important part of modern submarine to perform cruise, send and receive information, reach and leave the dock, observe and command [1,2]. Exposed to the

.Corresponding author: jijinzu@buaa.edu.cn 
surface of the sail, deck, bridge and outer walls of the lifting device, composing a large radar wave reflection source, can be discovered by the Army's synthetic aperture radar above sea level ranging from a few kilometers to $300 \mathrm{~km} \mathrm{[3].} \mathrm{The} \mathrm{sail} \mathrm{will} \mathrm{provide} \mathrm{a} \mathrm{clear}$ signal to the radar in a navigational or semi-submersible state. Therefore, reducing the size of the sail and optimizing the contour line is the goal pursued by submarine designers, aimed at reducing the adverse effect of the sail [4].

Considering the military requirements of the underwater non-acoustic stealth technology, several non-acoustic stealth individual technologies are emphasized based on foreign non-acoustic detection technology in reference [5]. The sails of SUBOFF submarine model with different horizontal position, height and shape are calculated in different schemes. The optimal design scheme on power performance is obtained by comprehensive comparison, guaranteeing the grid similarity in different cases [6]. Reference [7] have introduced the development of submarine stealth technology, then have analyzed the factors that affected the submarine stealth and put forward the technical measures to improve the stealth ability of submarine and the stealth ability of non-acoustic radiation. In the reference [8], the numerical simulation method is used to calculate the resistance, flow field and pressure distribution of the outer shell of different sails. The new type of attack submarines tend to be low-lying and increase the smooth transition between the hull and the sail, reducing the submarine hydrodynamic noise [9]. The mechanism, characteristics, evaluation methods of the sail noise and low noise design techniques of the sail are analyzed by Hu Qiwang, targeting shrinkage ratio sail model as the research subject [10]. The Renault average Navier-Stokes (RANS) method is used to calculate three dimensional viscous flow field of the submarine, in which the influence of the sail size and relative position on the hydrodynamic performance of the submarine is analyzed [11].

Since the 1960s, there have been a number of submarines with low-lying profile and excellent streamlined submarines, such as the German 212, the Swedish 2000 and the former Soviet Union's "AKULA" nuclear submarine. Relevant research has been carried out in the new 3D sail both at home and abroad. Gorski has designed and studied the new 3D sail and conducted a wind tunnel test on its flow field [12]. Wang Zhibo compared the influence of different three-dimensional sails on the wake field and analyzed the relationship between the sail configure and the characteristics of the wake field [13]. The influence of the three-dimensional dune-shaped sail on the resistance and wake field was analyzed, which verified that changing configure of the sail can reduce the resistance and achieve the uniform wake field [14].

Sufficient researches have been conducted on the traditional straight-walled sails and the new three-dimensional sails, but they focused on the properties of flow field of the sails. In this paper, the effect of three designed sails referring to AKULA, SUBOFF, VICTOR submarines on radar stealth will be compared, aimed at obtaining configure of good stealth performance. The three different styles of submarines are shown in Fig. 1. Fig. 1(a), Fig. 1(b) and Fig. 1(c), respectively, show the AKULA, SUBOFF and VICTOR submarine configuration characteristics.
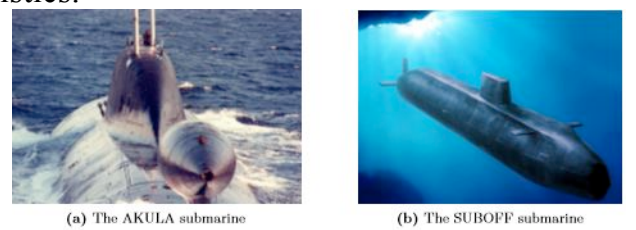

(b) The SUBOFF submarine

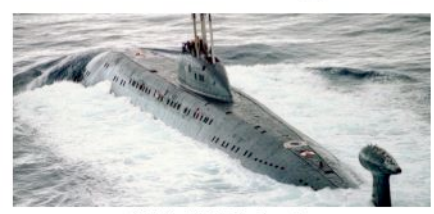

(c) The VICTOR submarine

Fig. 1. Three different styles of submarines 


\section{Theoretical basis}

The numerical simulation of a submarine sail's electromagnetic scattering characteristics includes the following two aspects: the pitch angle of the radar wave considering atmospheric refraction effect; the RCS calculation method.

\subsection{The pitch angle of the radar wave considering atmospheric refraction effect}

In the modern air-to-sea warfare, the largest radar threat to submarines is the enemy's airborne radar, compared to ground and sea based radar. Therefore, this paper assumes that the enemy radar is an airborne radar. And the electromagnetic scattering characteristics of the sail in various situations are discussed in this case. In calculating the target pitch angle, the radar wave in the Earth's atmospheric refraction effect is also taken into account. As is shown in Fig. 2, the target pitch angle is illustrated as follows:

$$
\theta_{1}=\arccos \left(\frac{a_{1}+h_{1}}{a_{1}+h_{t}} \cos \theta\right)
$$

Where $\theta_{1}$ is the target pitch angle, $\theta$ is the pitch angle of the airborne radar antenna, $a_{1}$ is the equivalent earth radius $\left(a_{1}=\frac{4}{3} a\right)$ and $\mathrm{a}$ is the radius of the earth $(\mathrm{a}=6370 \mathrm{~km}), h_{t}$ is the height of the airborne radar from the sea level, $h_{1}$ is the sea level height of the submarine measured from the sea surface, in which the earth is defined as a spherical surface with a radius of $6370 \mathrm{~km}$. The mentioned formula can be easily deduced using the sine theorem shown in Fig. 2

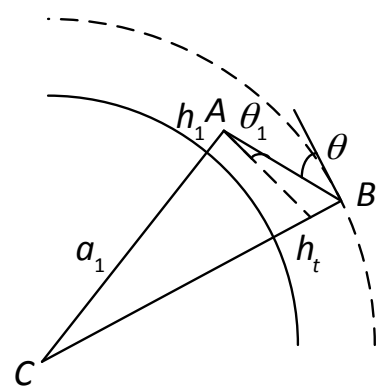

Fig. 2. The pitch angle of the sail relative to the radar wave

It can be seen that $\theta_{1}$ has a minimum value $\theta_{\min }$ in case that $\theta$ is infinitely close to zero. Whether a positive direction to increase or reduce in negative direction, the value of $\theta_{1}$ is obviously greater than $\theta_{\min }$. Thus effective pitch angle of the submarine can be acquired in face with airborne radar.

\subsection{RCS calculation method}

In this paper, the physical optics method and the equivalent electromagnetic flow method are used to simulate the electromagnetic scattering characteristics of the sail. Specifically, 
the physical optics method is used to calculate the surface facet scattering, while the equivalent electromagnetic flow method is used to calculate the edge diffraction [15].

The formula calculating the facet scattering by physical optics is as follows

$$
\sqrt{\sigma}=\frac{j k}{\sqrt{\pi}} \hat{n} \cdot\left(\hat{e}_{s} \times \hat{h}_{i}\right) \int_{S} e^{j k(\hat{s}-\hat{i}) \cdot \mathbf{r}^{\prime}} d S
$$

Where $\sigma$ is the RCS of a single surface element, $\mathrm{j}$ is an imaginary unit, $\mathrm{k}$ is the wave number, $\hat{n}$ is the surface normal vector, $\hat{e}_{s}$ is the direction of the antenna polarization, $\hat{h}_{i}$ is the direction of the incident magnetic field, $\hat{s}$ is the far field radiation direction, $\hat{i}$ is the incident direction, $\mathbf{r}^{\prime}$ is the source point coordinate, $d S$ is the surface element.

In the mentioned physical optics method, shadowing test is interpreted as two steps. First, facets are judged whether in the illuminated portion of the body. One effective method is that multiplying the incident direction by the normal direction of the facet. That is to say, if $\hat{n} \cdot \hat{i}<0$, the facet is illuminated. Second, in the illuminated region, one facet may be shadowed by another bright facet, which is taken into consideration in this paper. The idea is that when the center of a facet is shadowed by another facet, it is considered that the facet is shadowed $[16,17]$.

\section{Electromagnetic test validation}

This paper has researched the electromagnetic scattering characteristics of the radar cross section of the sails. According to the physical optics method and the equivalent electromagnetic flow method, the paper completes the simulation of the RCS characteristic of the sails. Before conducting the simulation, the accuracy and scientificity of the mentioned method should be validated by electromagnetic test.

\subsection{Electromagnetic test experiment}

In this paper, the 3D digital models of sails are created by CATIA software shown in Fig. 3. Then, the digital sail models are imported to the prepared program to simulate the sail's RCS obtaining the sail's electromagnetic scattering characteristics.

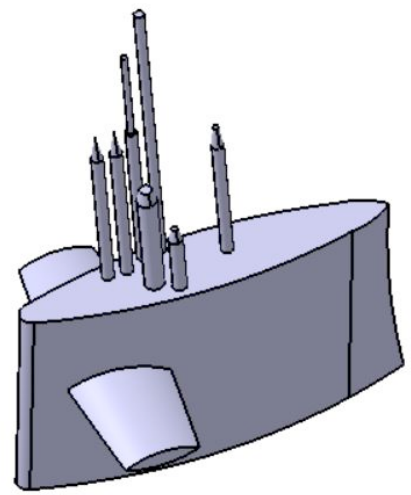

Fig. 3. 3D digital models of the submarine sail 
The 3D scale entity model of the sail is made and tested in an anechoic chamber as is shown in Fig. 4. Finally, the sail RCS curve of the electromagnetic test is acquired compared to the simulation shown in Fig. 5.

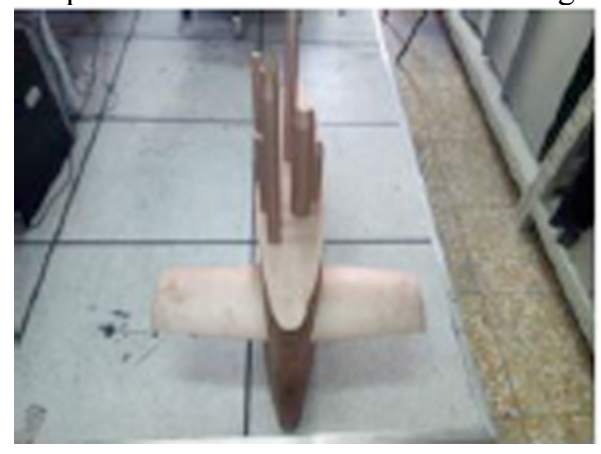

(a)Entity model of sail

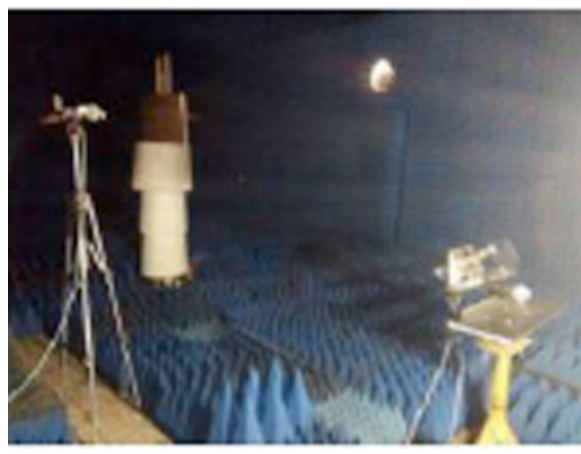

(b)The sail is tested

Fig. 4. Electromagnetic test of the sail in an anechoic chamber

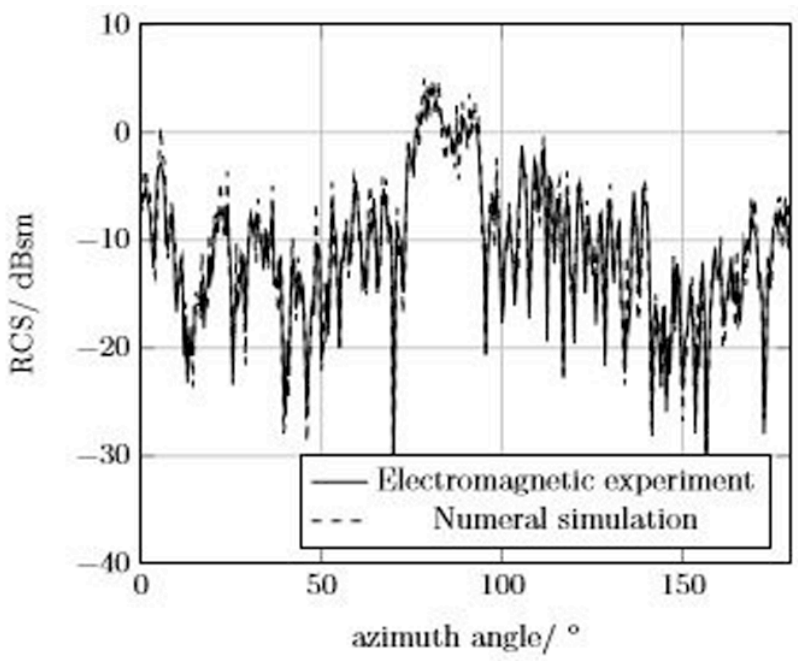

Fig. 5. RCS characteristics of the sail from simulation and electromagnetic test

\subsection{Result of electromagnetic test}

In this section, the program, based on the physical optics method and the equivalent electromagnetic method, is utilized to simulate the RCS of the 3D digital sail model. Then, the RCS characteristics of the electromagnetic test are compared with that obtained in the simulation program, as is shown in Fig. 5.

The initial conditions of the electromagnetic test and the numerical simulation remain the same. The incident radar wave is $X$ band, that is to say, the wavelength is $3 \mathrm{~cm}$. The incident wave pitch angle is $0^{\circ}$ and the polarization direction is horizontal polarization. Taking into account the symmetry, the azimuth is selected as $0^{\circ} \sim 180^{\circ}$. It can be seen from the Fig. 5 that the simulation results are in line with the electromagnetic test experiment. The results show that the numerical simulation method is accurate and scientific, which can be used to model more complex and large targets for electromagnetic scattering. 


\section{Sail RCS characteristics simulation and analysis}

\subsection{D models of sail}

In this section, on the background of the AKULA submarine, SUBOFF submarine and VICTOR submarine, CATIA software is employed to establish the 3D sail digital models shown in Fig. 6. These three typical different styles of submarine sails are created labelled as model A, B, C respectively. For the model A, the AKULA concept adopts a ternary complex surface presenting as smooth streamline along the $\mathrm{XY}, \mathrm{XZ}$ and $\mathrm{YZ}$ profile. The advantage of this configuration is to reduce the fluid resistance, improving the submarine's mobility, but the influence of stealth should be explored. For the model B, referring to SUBOFF submarine, it employs a conventional upright appearance, whose cross section is similar to the aircraft's airfoil. This is one of the most common and typical layouts, and most of the early submarine sails use this approach. The structural characteristics of Model $\mathrm{B}$ determine that it can accommodate more electronic and mechanical equipment. The model $\mathrm{C}$ can be simplified to be a combination of three simple rotating bodies (cone, cylinder and sphere), in line with the design idea of low-rise sails. Therefore, it can be regarded as a representative of the low-rise sails. Compared with Model B, Model A and Model $\mathrm{C}$ have a major advantage that the sail can be better fused with the hull.

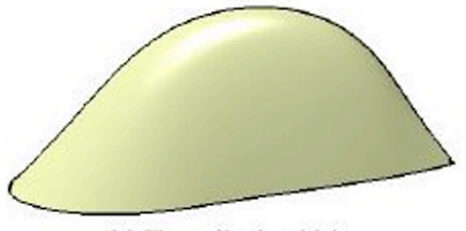

(a) The profile of model $\mathrm{A}$

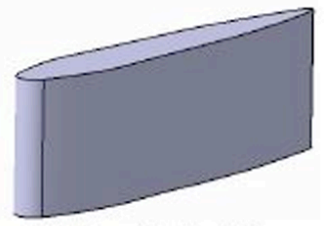

(b) The profile of model B

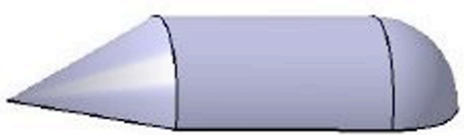

(c) The profile of model $\mathrm{C}$

Fig. 6. Three different styles of sails

The 3D sail model is simplified in accordance with the principle of preserving the main scattering characteristics and discarding those insignificant local features. In order to make the comparison results representative, the length of the first two models 1 , the maximum thickness $b$, and the height $h$ are consistent. The specific values are as follows: $1=14 \mathrm{~m}, b=$ $3 \mathrm{~m}, \mathrm{~h}=4 \mathrm{~m}$. The third model is a low-rise sail design, whose length and width are larger than the first two models and the height is lower than the first two. But the total volume is consistent with the first two sails.

\subsection{Sail RCS numerical simulation and analysis}

Based on the physical optics method and the equivalent electromagnetic flow method, the mentioned models are divided into multiple small triangular facets. When in calculation of the RCS of sails, the secondary reflection and shadowing need to be considered. It is generally believed that azimuth angle in the plane of the range of $0^{\circ} \sim 360^{\circ}$ belongs to the radar threat area of ships and submarines. Reference to the national naval airborne radar situation, the naval airborne radar frequency band is mostly $\mathrm{X}$-band $(8 \sim 12 \mathrm{GHz})$. Therefore, 
the RCS of sails are calculated at the middle value of the $\mathrm{X}$ band $(10 \mathrm{GHz})$ with the azimuth angle $0^{\circ} \sim 360^{\circ}$.

Taking into account the vertical polarization of the sea level scattering contribution is very large, the horizontal polarization is chosen in the simulation process. In this section, based on physical optics method and equivalent currents method, according to mentioned pitch angle range and radar band, MATLAB software is used to calculate the RCS of the previously established 3D sail models in Fig. 8. From the previous conclusion, the minimum pitch angle of the sail is $0.72^{\circ}$ relative to the airborne radar. In Fig. 8 , three curves represent the RCS of the three different styles of sails at the minimum pitch angle.

In Fig. 7, $\beta$ is denoted as the pitch angle of the sail. Fig. 9 shows the variation of the RCS at different azimuth angles with the pitch angle swinging between $1^{\circ} \sim 4^{\circ}$. As seen from the figure, with the pitch angle over a range, the AKULA RCS curve fluctuates but the number of spikes is always two. Moreover, the locations of the two spikes are concentrated in the vicinity of $103^{\circ}$ and $255^{\circ}$, almost without changing. The RCS curve is beneath $0 \mathrm{~dB}$ at most other angles, which conforms to stealth design principles. However, the RCS spike width becomes widened with the increase of the pitch angle. Meanwhile, the height of the spike increases with the increase of the elevation angle and then decreases. At the elevation angle of $3^{\circ}$, the RCS is as high as $49.98 \mathrm{~dB}$, which is $53.4 \%$ higher than when it is $1^{\circ}$.

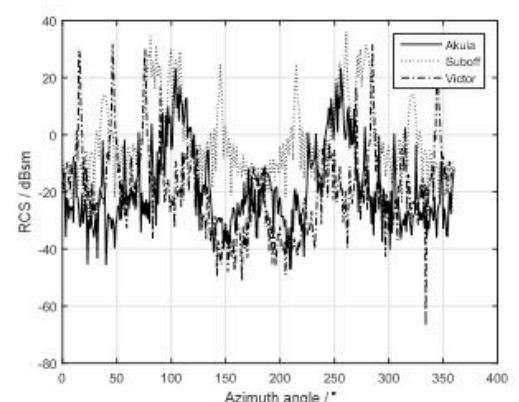

(a) $\beta=1^{\circ}$

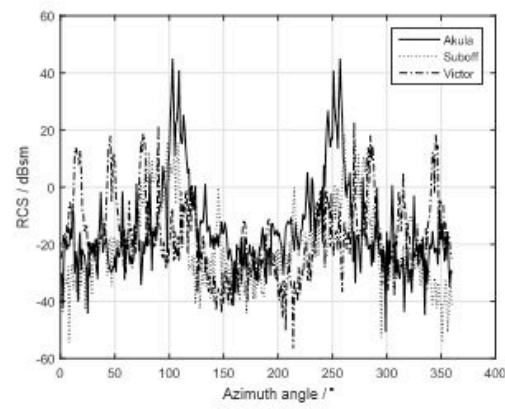

(c) $\beta=3^{\circ}$

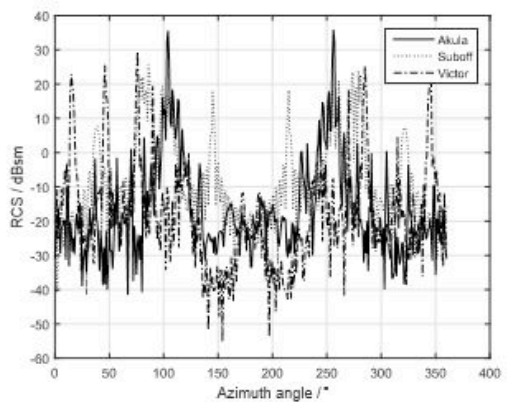

(b) $\beta=2^{\circ}$

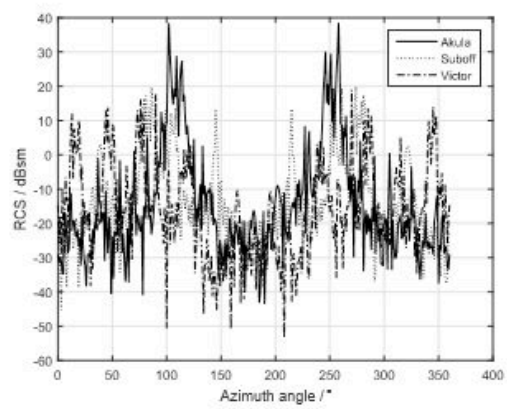

(d) $\beta=4^{\circ}$

Fig. 7. The comparison of the RCS results of the sails when $\beta=1 \sim 4^{\circ}$

With the variety of pitch angle, SUBOFF and VICTOR curves tend to remain essentially unchanged, but the heights of the curve spikes decrease. Compared with AKULA curve, the number of SUBOFF and VICTOR curve spikes is too much, whose 
peak values exceed $20 \mathrm{~dB}$ when it is $1^{\circ}$. This configuration design features lead to electromagnetic wave diffusing very strongly in a number of directions, which greatly increases the probability that the submarine will be detected by hostile radar.

Fig. 8 shows the mean RCS of the sails at $0 \sim 20^{\circ}$ pitch angle. The three curves representing AKULA, SUBOFF and VICTOR sails respectively are compared. The significance of mean RCS lies in that the probability of the sail being detected in all directions is assumed identical, in the face of airborne radar. Under this assumption, the influence of the pitch angle on the mean RCS of the sail is discussed. It can be seen from the Fig. 8, with the increase of the pitch angle, the AKULA mean RCS decreases first in a small range. Then, the curve tends to be stable and fluctuates very little. This indicates that the pitch angle change has little effect on the AKULA mean RCS. SUBOFF mean RCS curve shows a monotonically decreasing trend, but the decrease amplitude is gradually reduced. VICTOR's mean RCS curve goes up first and then the trend goes down, whose spike appears at $15^{\circ}$ pitch angle and its corresponding RCS is as high as $32.99 \mathrm{~dB}$.

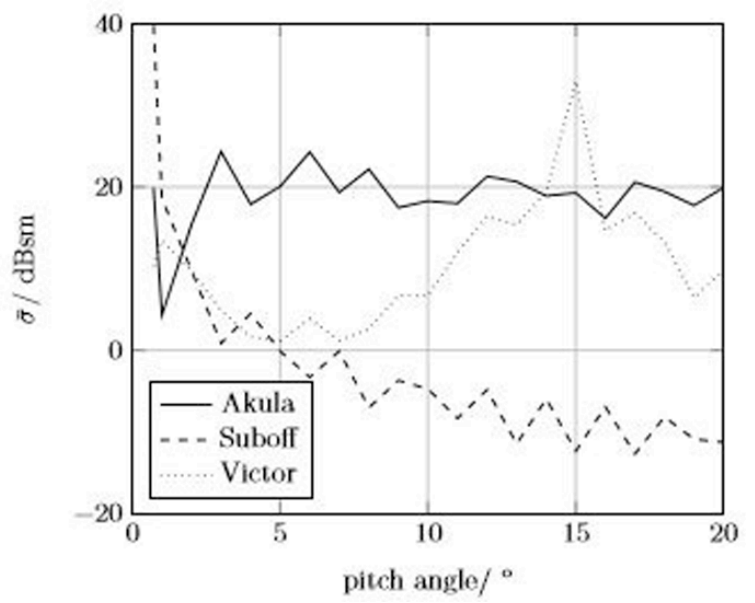

Fig. 8. The RCS mean curve of the sail with the pitch angle varies

\section{Conclusions}

The RCS characteristics of the AKULA sail, SUBOFF sail and VICTOR sail on the water have been calculated and analyzed in this paper. It is assumed that the threat to the sails is airborne radar. Based on the concept of the fusion of the sail and the hull, the AKULA model is created. Moreover, on the basis of the concept of low-lying sail designing, the VICTOR model is built. Both of them are compared with the SUBOFF model representing the traditional sails. Utilizing the physical optics method and the equivalent electromagnetic flow method, the RCS characteristics of three designed sails have been calculated under X band of radar in this paper. And the mean RCS of the sails are also investigated with the change of pitch angle. Then, a sail RCS reduction strategy on the stealth configuration of the subsequent modification is proposed. Through the mentioned research, the conclusions of this paper can be summarized as follows:

1. Under $0.7179^{\circ} \sim 4^{\circ}$ pitch angle, the AKULA RCS curve has only two spikes, whose spikes are concentrated in the vicinity of $103^{\circ}$ and $255^{\circ}$. Furthermore, the scattering direction is non-major threat direction, which is highly advantageous for monostatic stealth. Compared to AKULA, the other two have too much spikes on RCS curves resulting in the probability of being detected by enemy radar increasing greatly. 
Therefore, the AKULA sail is considerably superior to SUBOFF sail and VICTOR sail on stealth performance with the pitch angle less than $4^{\circ}$. According to the AKULA electromagnetic scattering characteristics, an effective measure to improve the stealth effect of the AKULA sail is to apply the absorbing material in the vicinity of $103^{\circ}$ and $255^{\circ}$ azimuth angle to consume the scattered energy of the position.

2. Observing the mean RCS curve, when the pitch angle exceeds $10^{\circ}$, the SUBOFF mean RCS is more than $50 \%$ lower than the other two. It is indicated that the SUBOFF sail can be given priority in the high pitch angle.

\section{References}

1. Roy Burcher, Louis Rydill, and C. W Bert. Concepts in submarine design. Journal of Applied Mechanics, 62(1), 1995.

2. Yu Cun Pan, Qi Dou Zhou, and Huai Xin Zhang. Numerical simulation of rotating arm test for prediction of submarine rotary derivatives. Journal of Hydrodynamics Ser B, 27(1):68-75, 2015.

3. Yang Jian, $\mathrm{Wu} \mathrm{Chao}$, and $\mathrm{Gu}$ Yang. Measures to improve the stealth capability of submarine. Shipbuilding Technology, (1):22-24, 2006.

4. Du Bo, Huang Jianwei, and Chen Yuan. Optimal design of the top form of submarine sail. Ship and ocean engineer, 36(2):107-110, 2007.

5. Peng Liang, Wang Jianxun, and Deng Haihua. Review of the non-acoustic detection and stealth technology. Ship Science and technology, 36(5):6-10, 2014.

6. Lv Minghe. Optimization design of submarine command platform enclosure. China water and transport, 14(1):1-4, 2014.

7. Jiao Fangjin. Development of submarine stealth technology. National defense science and technology, (4):35-37, 2003.

8. Huilan Yao, Huaixin Zhang, Hutao Liu, and Wencheng Jiang. Numerical study of flow-excited noise of a submarine with full appendages considering fluid structure interaction using the boundary element method. Engineering Analysis with Boundary Elements, 77:1-9, 2017.

9. Chen Feng and Qi Guoying. Research on technical characteristic of foreign new generation nuclear submarine. Ship science and technology, 36(9):153-157, 2014.

10. $\mathrm{Hu}$ Qiwang. Research of noise mechanisms of submarine sail model. PhD thesis, Harbin Engineering University, 2007.

11. Wu Fangliang, Wu Xiaoguang, and Ma Yunyi. The effect of submarine sail on the resistance and wake field. The Ocean Engineering, 27(3):91-99, 2009.

12. Joseph J. Gorski and Roderick M. Coleman. Use of rans calculations in the design of a submarine sail. 2003.

13. Zhibo Wang, Huizhi Yao, and Nan Zhang. Calculation and analysis of the effects of sail form on submarine wake. Journal of Ship Mechanics, 2009.

14. Longju Liu and Yong $\mathrm{Xu}$. Numerical study about the effect of touradon shaped submarine sail on flow field. Journal of Sichuan Ordnance, 2014.

15. George T Ruck. Radar cross section handbook, volume 2. Plenum Publishing Corporation, 1970.

16. Jinzu Ji and Hongqian $\mathrm{Wu}$. Shadowing algorithm of facets in physical optics (po) based on determinant. Optik - International Journal for Light and Electron Optics, 126(14):1366-1368, 2015.

17. Ji Jinzu and Liu Zhanhe. Electromagnetic occultation algorithm based on facets grouping and optimization. Journal of Beijing University of Aeronautics and Astronautics, 35(4):453-456, 2009. 